\title{
Inmigración y servicios sociales
}

\author{
Carmen Alemán Bracho \\ UNED. Facultad de Ciencias Políticas y Sociología \\ caleman@der.uned.es
}

Recibido: 14-10-2010

Aceptado: 02-06-2011

\section{Resumen}

El artículo analiza los efectos de la crisis económica en el desenvolvimiento de los servicios sociales. Desde la Constitución, los ayuntamientos y las comunidades autónomas han dado un gran impulso a los servicios sociales, de modo que lo han convertido en un sector muy dinámico y expansivo. Por eso ha tenido que adaptarse a un fenómeno como la inmigración, novedoso en España y con unas características (muy rápida, poco regulada, muy concentrada en los lugares de destino, etc.) que han dificultado su gestión. La crisis económica afecta muy negativamente a los inmigrantes, entre otras cosas, porque son mayoría en los sectores más afectados por la crisis, como la construcción, porque carecen de una red de apoyos familiares en España y porque mantienen obligaciones económicas con sus países de origen, de difícil cumplimiento en situaciones de paro. Como consecuencia, sus demandas de servicios y ayudas van a multiplicarse, van a ser más heterogéneas y a prolongarse durante más tiempo. La incertidumbre reside en pronosticar si las administraciones optarán por aumentar la actual calidad de las prestaciones y los servicios, mantenerla o reducirla. Un pronóstico cuya respuesta puede ser muy diferente si la crisis dura unos cuantos años, y puede variar en los diferentes servicios y en las diferentes comunidades autónomas, cada vez más diversas en sus políticas en este área.

Palabras clave: integración social; política social; redes sociales; España.

\section{Abstract. Immigration and social services}

This article examines the possible impact of the current economic crisis on the development of the social services. Since the signing of the Spanish constitution, town councils and Autonomous Communities have given a boost to social services, which have become a dynamic and expanding sector. As such, they have had to quickly adapt to the phenomenon of immigration, a novelty in Spain and with certain characteristics (very rapid, underregulated, and concentrated in certain locations) that have made its management more difficult. The economic crisis has had very serious effects on immigrants because, among other reasons, they form a majority in some of the sectors worst-affected by the crisis, like the construction industry, because immigrants sometimes lack a family support structure 
in Spain and they continue to have financial obligations in their countries of origin, which are difficult to meet if they are unemployed. As a result of this, their demands on social services will grow sharply, becoming more heterogeneous and lasting longer. It remains uncertain whether the Administration will opt for improving the quality of social services, keeping it at the same level, or reducing it. The answer will depend on the length of the crisis, on the type of service, and on the positioning of the different Autonomous Communities, which tend to have different policies in this matter.

Key words: social integration; social policies; social networks; Spain.

\author{
Sumario \\ 1. Introducción 4. Recursos sociales para el bienestar \\ 2. Las dificultades estructurales \\ social de los inmigrantes \\ 3. El crecimiento de las demandas \\ 5. Conclusión \\ 6. Referencias bibliográficas
}

\title{
1. Introducción
}

España es un país con larga tradición en movimientos de población, voluntarios y forzosos. Exilios, falta de trabajo y pobreza han sido motores muy potentes de los continuos desplazamientos de gran número de ciudadanos hacia los más diversos lugares. $\mathrm{Si}$, en las primeras décadas del siglo $\mathrm{xx}$, el destino mayoritario de la emigración socioeconómica fue con carácter definitivo a América del Sur, con posterioridad se frena y desaparece casi en su totalidad.

El fin de la Guerra Civil (1939) produce un gran contingente de refugiados políticos, pero los posteriores controles estatales y la guerra mundial ocasionaron la disminución y luego la práctica desaparición de los movimientos de población hacia ese destino. El nuevo flujo va a dirigirse años después, muy mayoritariamente, hacia los países europeos desarrollados. Entre las décadas de 1960 i 1970, hasta la crisis del petróleo de 1973, un gran número de españoles emigran en su mayoría con voluntad de retorno. El punto álgido de esa emigración fue el año 1964, en el que se estima salieron 300.000 personas. Una parte importante del despegue económico de España en esos años se debe a las aportaciones de los emigrantes españoles en Europa, por su aportación de divisas en monedas fuertes.

En este escenario es preciso situar el desarrollo de los servicios sociales. $\mathrm{Si}$ los servicios sociales tienen en España una corta historia, más reducida aún es la que se refiere a la inmigración. De hecho, la primera experiencia y actividad de los servicios sociales después de la restauración democrática fue la atención a los emigrantes españoles que retornaban a España, sobre todo de países democráticos europeos, como consecuencia de la crisis económica de 1973 y el paro subsiguiente. En muchos casos, su retorno como emigrantes parados significó la puesta en marcha de instalaciones, equipamientos y servicios de atención 
personal. Y en ello tuvieron protagonismo relevante los nuevos ayuntamientos democráticos.

Las últimas décadas del siglo $\mathrm{xx}$ dieron un giro completo a los movimientos de población en España, puesto que se rompió por completo con tendencias anteriores. Por una parte, se detuvo casi de manera total la emigración exterior. La crisis económica hizo desaparecer las oportunidades de empleo. Por otra parte, los nuevos ayuntamientos democráticos comenzaron a mejorar los equipamientos, los servicios y la calidad de vida de los pueblos, lo cual favoreció la retención de la población en ellos. Esta dinámica se vio favorecida igualmente por otras medidas del poder central y de los nuevos responsables autonómicos, que desarrollaron una nuevas ayudas de política social y subsidios a la población en paro, implantadas entonces.

Pero algo semejante, y por las mismas razones, ocurrió con los servicios sociales simultáneamente. Desde la década de 1970 hasta nuestros días, las innovaciones en los servicios sociales han sido permanentes y en todas las áreas: en la ordenación del sector, en la legislación, en el reclutamiento de personal y en la implicación de los diferentes ayuntamientos en su desarrollo y potenciación. En la primera década del siglo Xxi, los servicios sociales tienen que afrontar, sin embargo, un escenario muy diverso. Tres de sus rasgos deben mencionarse. En primer lugar, han de hacer frente a una aguda crisis económica y, por ello, en segundo lugar, a un incremento de demandas y necesidades de los ciudadanos, y, en tercer lugar, una mayor diversidad de demandas o incluso la aparición de demandas o necesidades novedosas. Las dificultades presupuestarias de las administraciones públicas constituye un escenario complicado para la respuesta institucional al previsible incremento de las demandas.

Examinar las relaciones entre los servicios sociales y la inmigración es algo particularmente oportuno, porque la dinámica social ha obligado a realizar un giro importante en la regulación de este sector. La configuración de los servicios sociales se realizó a partir de la Constitución, pero, dado que la Constitución atribuye al Estado la competencia en inmigración, emigración, extranjería y derecho de asilo y la competencia en asistencia social a las comunidades autónomas, fue desde la aprobación de la Constitución, y de las diferentes leyes de servicios sociales en las comunidades cuando quedaron distribuidas las competencias en este ámbito. El tratamiento de la inmigración ha variado sustancialmente, como consecuencia del incremento de su frecuencia en España. La regulación del acceso de los inmigrantes a los servicios sociales en las leyes autonómicas ha sido sintetizada muy bien por Pilar Andrés (2004), quien diferencia tres fases:

1. A partir de 1982, con las primeras leyes autonómicas. En estas leyes, una parte de las cuales continúa todavía en vigor, no se contempla el fenómeno de la inmigración.

2. En las leyes de servicios sociales de la segunda mitad de la década de 1990. Éstas recogen las nuevas realidades sociales y, bajo el influjo de las nuevas 
realidades legales sobre extranjería, se reconoce el acceso a los servicios sociales como un derecho básico para todas las personas, puesto que desaparece la referencia a la nacionalidad española como requisito.

3. Leyes promulgadas a partir del año 2000. Se aprueban después de las nuevas leyes de extranjería y el crecimiento de la inmigración en España. Incluye a normas aprobadas en Asturias, Cataluña, Murcia, La Rioja y País Vasco. En ellas ya se encuentran tratamientos muy explícitos sobre la inmigración. Así, se reconocen como titulares de derecho a todos los ciudadanos residentes o transeúntes (La Rioja), o a los nacionales de los estados miembros de la Unión Europea empadronados y a quienes, no siendo miembros de esos estados, se encuentren en la comunidad autónoma (Asturias), y se comprometen a aprobar un catálogo de prestaciones, algunas de las cuales tendrán carácter de fundamentales y serán exigibles como derecho subjetivo. O el caso de la Ley de Murcia, que contempla explícitamente que uno de los sectores de los servicios sociales esté especializado en los inmigrantes.

En términos generales, por tanto, la legislación vigente recoge una concepción muy amplia del derecho de acceso a los servicios sociales comunitarios y a sus recursos para todos los extranjeros, independientemente de su situación administrativa, pero que han cumplido con el requisito de empadronamiento, puesto que, para acceder a las prestaciones económicas — con diversas salvedades-, se requiere la residencia legal (véase también Andrés Juste, 2004).

Pero las leyes no son toda la realidad, y la inmigración española presenta peculiaridades respecto a la experiencia de otros países europeos. Soriano las ha sintetizado con precisión:

Se ha operado en este campo sin experiencia previa, teniendo que abordar el problema sin política pública nacional de referencia, sin instrumentos de gestión rutinizados, sin cultura de inmigración y, por tanto, sin nociones precisas acerca de los impactos sociales que pudiera causar la inmigración, y las medidas gubernamentales para conducirlas. Se desconocía el problema, sus posibles dimensiones, los instrumentos para hacerles frente y la propia capacidad de la sociedad y la economía española para absorber un flujo de inmigración. (Soriano, 2004: 339)

Las leyes, pues, han evolucionado con rapidez, intentando dar respuesta a la nueva realidad inmigratoria en España. Y no resulta fácil sintetizar las singularidades que la actual situación de crisis económica mundial, con sus peculiaridades en España, puede originar entre la población inmigrante y sus repercusiones previsibles en los servicios sociales. Aunque dejemos para los economistas el examen de la crisis económica y sus especificidades, sí es necesario realizar algunas precisiones para comprender su alcance en los servicios sociales para los inmigrantes. 


\section{Las dificultades estructurales}

Las singularidades del proceso inmigratorio en España afectan a la capacidad disponible de los servicios sociales para prestar eficazmente sus asistencias. Algunas características del proceso dificultan objetivamente la prestación de servicios adecuados en muchas ocasiones. La inmigración ha sido en España:

1. Un fenómeno muy reciente, que puede situarse en la década de 1980 , lo cual quiere decir que han debido improvisarse los mecanismos y los organismos de acogida de los inmigrantes en dos décadas. El crecimiento de la inmigración ha sido vertiginoso. En diez años, la población extranjera se ha multiplicado en más de ocho veces, de modo que ha pasado de ser 637.085 en 1998, lo que suponía el 1,6\% del total de la población en España, a 5.220.577 personas en 2008, que significa el 11,3\% del total (Moreno y Aierdi, 2008: 8). El rápido crecimiento de la inmigración tiene importantes consecuencias para los servicios sociales. Como consecuencia de ese ritmo tan rápido, la mayoría de los inmigrantes llevan pocos años de residencia en España y, por consiguiente, es previsible que no hayan concluido su proceso de integración social y mantengan necesidades y demandas superiores a quienes llevan más años en nuestro país.

2. Un movimiento muy escasamente controlado en el acceso, lo que ha favorecido el auge de mafias, las condiciones deplorables de entrada en España (en pateras u otros medios de transporte, en los cuales, en muchos casos, los inmigrantes arriesgan su vida), con dependencia posterior de los sectores que intervinieron para organizar su entrada. Una situación que ha generado durante años dependencias económicas y aún personales de esas mafias, lo cual ha obstaculizado el posterior proceso de integración.

3. Se ha producido una muy alta concentración de inmigrantes en determinadas comunidades, municipios o, incluso, barrios, que generan demandas muy altas de servicios sociales. Las disparidades entre comunidades autónomas son muy acusadas. Las comunidades que cuentan con un porcentaje más alto de extranjeros en relación con su población son: Cataluña, Baleares, Madrid, Comunidad Valenciana, Murcia y Canarias. Y, dentro de ellas, existe también muy alta concentración en determinados municipios.

4. Las dificultades financieras de los ayuntamientos para responder a las necesidades generadas por la concentración de inmigrantes; unas dificultades acrecentadas por las relaciones, nunca nítidas, entre las comunidades autónomas - con competencia legal en servicios sociales - y los ayuntamientos destinatarios inmediatos de las demandas.

5. La creciente heterogeneidad de los servicios sociales en España, resultado de la competencia autonómica y la existencia de diecisiete sistemas — cada uno con sus propias leyes - organizativos, de prestaciones y objetivos. Los estudios realizados por el BBVA sobre las políticas autonómicas en materia inmigratoria evidencian esta diversidad (Allemán et al., 2009). 
Por otra parte, la presencia foránea en España es muy heterogénea y no siempre se diferencia con precisión. Extranjero designa a aquellas personas que no tienen la nacionalidad española, lo que genera derechos diferenciales respecto a los nacionales. Pero un extranjero puede dejar de serlo mediante la nacionalización, que es la adquisición de una nacionalidad diferente a la de nacimiento (por adopción, por matrimonio, por concesión extraordinaria, etc.), en nuestro caso la española. Refugiado define la situación de la persona que abandona un país y se incorpora a otro, incluso sin documentos, por razones humanitarias, de guerra o persecuciones. Irregular alude a la situación administrativa de carencia de documentos exigidos en un país para residir o trabajar. E inmigrante se refiere a la persona que se desplaza de un país a otro por razones económicas o de empleo.

La nacionalización tiene una repercusión considerable en la magnitud del fenómeno inmigratorio. En España es lo que está ocurriendo. La nacionalización crece a mayor ritmo incluso que la inmigración, las estadísticas disponibles así lo demuestran. Casi 300.000 personas han sido nacionalizadas en siete años, de modo que el incremento acumulado asciende al 300\%. Los ecuatorianos y los colombianos son quienes obtienen más nacionalizaciones. En conjunto, los iberoamericanos suponen el $72 \%$ del total de concesiones. Pero otros países cuentan también con altos porcentajes; por ejemplo, en seis años se ha multiplicado por $2,8 \%$ el número de marroquíes que obtienen la nacionalidad española. Los motivos de la concesión sufren ligeros cambios en estos años, pero sustancialmente se mantienen de mayor a menor frecuencia las siguientes causas:

1. Residencia durante dos años.

2. Matrimonio con un español o una española.

3. Diez años de residencia.

4. Nacimiento en España.

5. Hijo o nieto de español de origen.

Este creciente porcentaje de población nacionalizada en España no puede incluirse cuando se menciona la inmigración. No aparece en las estadísticas de

Tabla 1. Concesiones de nacionalidad

\begin{tabular}{lccc}
\hline & & \multicolumn{2}{c}{ Variacion anual } \\
\cline { 3 - 4 } Año & Número & Absoluta & $\%$ \\
2001 & 16.743 & - & - \\
2002 & 21.810 & 5.067 & 30,3 \\
2003 & 26.556 & 4.746 & 21,8 \\
2004 & 38.335 & 11.779 & 44,4 \\
2005 & 42.829 & 4.494 & 11,7 \\
2006 & 62.339 & 19.510 & 45,6 \\
2007 & 71.810 & 9.471 & 15,2 \\
\hline
\end{tabular}

Fuente: Boletín Estadístico de Extranjería e Inmigración, 18, noviembre de 2008. 
inmigración, porque ha dejado formalmente de serlo. Sin duda, la obtención de la nacionalidad es un indicador de integración social muy relevante, pero no puede interpretarse como la desaparición de todas las dificultades de su existencia en su nueva ciudadanía. Este instrumento legal no impide que dichos nuevos españoles mantengan de hecho, en algunos casos, pautas, tendencias e incluso problemas propios de los inmigrantes que no han cambiado de nacionalidad. Sus problemas pueden ser más parecidos a los de las personas de sus países de origen que a los de sus nuevos conciudadanos españoles.

La respuesta estatal al nuevo fenómeno de la inmigración ha sido cambiante y dubitativa en algunas ocasiones, aunque ha tenido una creciente vinculación con otros países de la Unión Europea. Una vía de acción ha sido, desde luego, la elevación del rango en la Administración con competencias en la materia. Si existía una secretaría de Estado, en 2008, en la Administración central, se crea el Ministerio de Trabajo e Inmigración, siguiendo la pauta establecida en diversas comunidades autónomas que ya incluían la denominación de inmigración en sus consejerías.

La presencia de la inmigración en los máximos órganos administrativos evidencia la relevancia de la cuestión en la agenda pública. En pocos años, pasó de ser una cuestión ignorada a constituir uno de los temas considerados más importantes por la opinión pública. La densidad administrativa — evidenciada por la proliferación de comisiones interministeriales, foros para la integración social de los inmigrantes, observatorios de xenofobia y racismo, como entes de distinta naturaleza de coordinación entre las comunidades y entre las entidades locales-, acredita la centralidad política adquirida por la inmigración en una década en España.

Esta centralidad ha venido acompañada por la proliferación normativa. Atendiendo sólo a la aprobada por la Administración central, sometida a numerosas modificaciones y, lo que es más relevante, a la ampliación de sus objetivos, conviene mencionar la Ley Orgánica sobre derechos y libertades de los extranjeros en España y su integración social, de 2000. La Ley dedica una atención especial al acceso de los inmigrantes a los servicios públicos de bienestar atendiendo a la educación, el trabajo, la Seguridad Social, la asistencia sanitaria, la vivienda y los servicios sociales (Alemán y García Serrano, 2008: 299 y s.). Con posterioridad, ha sido modificada de manera sustancial en repetidas ocasiones, para responder a la naturaleza cambiante del fenómeno y para adaptarse a las directivas de la Unión Europea y a las sentencias del Tribunal Supremo y del Tribunal Constitucional. Merecen citarse las sentencias del Tribunal Constitucional 236/2007, de 7 de noviembre, y la de 259/2007, de 19 de diciembre, que declararon inconstitucionales algunos artículos de la Ley que no reconocían el ejercicio de determinados derechos de participación (reunión, manifestación, asociación, sindicación y huelga) a los extranjeros en situación de irregularidad administrativa e instaban al legislador a proceder a la reforma.

La amplitud de los objetivos dirigidos a la inmigración se observa sobre todo en los diferentes planes de integración de ámbito estatal. Entre 2000 y 
2004, con el Programa Global de Regulación y Coordinación de Extranjería e Inmigración (Plan Greco), se había puesto de relieve la necesidad del trabajo de los inmigrantes para la economía española, relacionaba los objetivos de las políticas de integración y ordenaba las medidas a adoptar por parte de los diferentes organismos de la Administración central. El vigente Plan Estratégico de Ciudadanía e Integración, dirigido al conjunto de la población, tanto autóctona como inmigrante. Su objetivo es fortalecer la cohesión social mediante políticas públicas basadas en la igualdad de derechos y deberes, la igualdad de oportunidades, el desarrollo de un sentimiento de pertenencia de la población inmigrada a la sociedad española y el respeto a la diversidad. El Plan Estratégico se divide en doce áreas, entre las cuales encontramos las de acogida, educación, vivienda, salud, juventud e infancia, mujer y servicios sociales.

Con todos los cambios que se han producido, de manera sintética, puede afirmarse que las políticas públicas en España han desarrollado sobre todo cuatro líneas de actuación (Alemán y García Serrano, 2008):

1. La lucha contra la inmigración irregular y el control de flujos.

2. La integración de la población inmigrante.

3. La vinculación entre migración y mercado de trabajo.

4. Una tendencia con creciente relevancia: las políticas de cooperación y desarrollo con los países de procedencia de inmigrantes, sobre todo con los de África.

La creciente cooperación europea en materia de inmigración es un hecho. Se ha pasado de la cooperación intergubernamental a los primeros pasos de una política común de inmigración y asilo, y del acento en el control de fronteras a la inclusión de la integración como parte de la agenda sobre inmigración de la Unión Europea. Ese es, desde luego, el objetivo, aunque los resultados no siempre están a la altura de los deseos o las finalidades.

El crecimiento de la inmigración y la proliferación normativa en este campo ha venido acompañada por la modificación de objetivos. Ahora el propósito preferente es la integración. Ello significará cada vez más que muchos extranjeros comiencen a acudir a los servicios sociales generales para demandar prestaciones cuyo objetivo, más o menos explícito, es la integración y la normalización en nuestra sociedad (ayudas de libros para menores, ayudas de comedor en colegios, ayudas para alquiler de vivienda y otros muchos apoyos que recibe la población autóctona). Los extranjeros que vinieron a nuestro país en los primeros años de la década de 1990 comenzaron a reagrupar la familia, ya que su situación personal se estabilizaba. Así, pues, los miembros familiares que estaban en los países de origen comenzaron a reencontrarse en el nuevo país. Al mismo tiempo, se produce una emigración con el conjunto de la unidad familiar. El resultado de esta nueva situación es evidente: comienza a producirse un progresivo incremento de extranjeros en la red de servicios sociales generales (Guillén et al., 2001: 235).

Las transformaciones en la inmigración son importantes. Contrariamente al estereotipo que muchas veces se difunde, que presenta al inmigrante como 
analfabeto y con carencias importantes en educación, su situación de vulnerabilidad proviene de su precaria situación económica; de su inestable situación laboral, a veces como consecuencia de su situación de irregularidad, y de las barreras lingüísticas y culturales a las que se enfrenta (Montagud y Torres, 2002: 25).

\section{El crecimiento de las demandas}

El examen de la evolución de las demandas de servicios sociales por parte de la población inmigrante viene condicionado por la perspectiva cualitativa que se busque o que se espere. No debe olvidarse que la situación de los servicios sociales depende de manera genérica de las leyes autonómicas, también es importante el papel que desempeñan las leyes autonómicas del salario social y, en la práctica, de las decisiones y políticas de los gobiernos central y autonómico y de los ayuntamientos. Por consiguiente, la trayectoria futura vendrá determinada, anualmente, por un gran número de decisiones y orientaciones políticas muy atomizadas en España. Resulta imposible establecer previsiones en este escenario real, pero eso no impide afirmar que, en la situación de crisis, las decisiones y, lo que es más decisivo, los recursos pueden dirigirse hacia tres objetivos muy diferentes. Se trata de tres alternativas hacia las que pueden orientarse las estrategias públicas de los servicios sociales, donde pueden establecerse tres objetivos.

Un primer posible propósito sería establecer como objetivo mejorar la calidad, en tal caso, los servicios sociales deberán convertirse en el futuro inmediato, en instituciones prioritarias por parte de las administraciones públicas, con un importante crecimiento de sus dotaciones presupuestarias y prioridad institucional. Una hipótesis poco probable, al menos con carácter general en España. Otro segundo objetivo posible es el de mantener la calidad actual de los servicios sociales. Se trata de un escenario más realista que el anterior, pero que va a requerir también incrementos en las dotaciones presupuestarias, porque la situación de crisis generará mayores demandas, más heterogéneas y mayor número de usuarios de los servicios sociales, por lo que el mantenimiento de la calidad hará necesario reforzar las dotaciones de que dispongan. Y el tercer objetivo hipotético, pero posible, sería aceptar la pérdida de calidad que actualmente ofrecen los servicios sociales, vía reducción de personal o por el recorte de prestaciones, servicios y apoyos. Se trata, sin duda, de la opción menos previsible, por lo menos no parece posible que llegue a plantearse explícita y formalmente; lo cual, sin embargo, no excluye que, en algunos casos concretos - sea en alguna comunidad autónoma o en algún programa delimitado-, pueda ocurrir en la práctica con mecanismos más o menos discretos, incluso jugando con el factor tiempo, como retrasando inversiones o ralentizando el ritmo de construcciones o de contrataciones, etc.

La situación real puede ser extraordinariamente diversa en España. Si la crisis dura varios años, las comunidades autónomas, los ayuntamientos y la Administración central pueden cambiar sus posiciones, y además pueden gra- 
duar sus políticas en función de las diferentes necesidades que se les presenten. Una realidad que diversificará aún más la situación de los servicios sociales en España.

No puede excluirse por completo, sin embargo, que la crisis conduzca a otras dos alternativas posibles: puede reforzar el compromiso de las administraciones públicas en la prestación de los servicios, pero también puede evolucionar en sentido contrario, incrementando el protagonismo de otras organizaciones - muy particularmente las $\mathrm{ONG}$ —en la prestación de servicios sociales en España. Y esta disyuntiva -Administración pública versus ONGpuede plantearse de manera global o total, o bien de manera sectorial, es decir, en alguna actividad en concreto.

Todo indica que la atención a los inmigrantes es una actividad que cuenta ya hoy con un importante protagonismo de sectores ajenos a las administraciones públicas, aunque dispongan de su financiación y respaldo. Las ONG han adquirido gran relevancia y probablemente la incrementen en el futuro inmediato, puesto que la relación con los inmigrantes se hace mucho más fluida que con las dependencias administrativas en muchas ocasiones, sobre todo por parte de los irregulares, quienes acuden con menos temores o suspicacias a las ONG que a los organismos públicos. De todas formas, la Administración pública continuará manteniendo el liderazgo en situación de crisis de los servicios sociales, de manera directa y también como soporte financiero de gran número de programas para inmigrantes de las $\mathrm{ONG}$.

La población inmigrante va a ser más propensa que la población general a necesitar apoyos sociales, debido a tres rasgos singulares de su situación en España. Por una parte, su presencia está muy concentrada en sectores con alta incidencia de la temporalidad — como la construcción—, muy afectada por la destrucción de empleo y muy sensibles a las incidencias del ciclo económico - como la hostelería - y, por consiguiente, crece rápidamente el paro en este sector. En segundo lugar, los inmigrantes carecen con gran frecuencia de la red de soportes familiares que para los españoles fue la principal base de apoyo y supervivencia en la crisis de empleo de las décadas de 1970 y 1980. Una aportación con que la mayor parte de los inmigrantes no podrá contar en España. $\mathrm{Y}$, en tercer lugar, gran parte de los inmigrantes ha de hacer frente también a las necesidades generadas por su red familiar en sus países de origen, en buena medida sustentados desde las aportaciones enviadas desde España.

La crisis económica va a cortar sustantivamente ese flujo económico. Las estadísticas del Banco de España de las transferencias de los inmigrantes recogen el brusco y rápido descenso desde hace meses de esas aportaciones, cuyo disminución se acelera. Y, probablemente, las dificultades de adaptarse a la actual situación se incrementan, debido a que se producen después de un rápido crecimiento de las transferencias, con la consiguiente movilización de decisiones e inversiones en los países de origen.

En este escenario de crisis, los inmigrantes se encuentran ante la necesidad de hacer frente a sus propias necesidades en España, además de las peticiones de transferencias a sus países de procedencia, para hacer frente a las estrategias 
Tabla 2. Envíos de remesas de emigrantes desde España (datos en millones de euros)

\begin{tabular}{|c|c|c|c|c|c|}
\hline Año & $1{ }^{\text {er }}$ trimestre & $2 .^{\circ}$ trimestre & $3 .^{\text {er }}$ trimestre & $4 .^{\circ}$ trimestre & Total \\
\hline 2005 & - & 1.142 & 1.307 & 1.407 & 4.189 \\
\hline 2006 & 1.473 & 1.712 & 1.808 & 2.066 & 4.938 \\
\hline 2007 & 1.908 & 2.011 & 2.210 & 2.316 & 8.445 \\
\hline 2008 & 1.998 & 1.928 & 2.075 & 1.839 & 7.840 \\
\hline 2009 & 1.654 & - & - & - & - \\
\hline
\end{tabular}

Fuente: Banco de España. Boletín Estadístico. Balanza de Pagos. Transferencias Corrientes. Pagos: Remesas de Trabajadores.

personales o familiares en preparación para el presente o el futuro (el pago de costes económicos para una vivienda o un negocio o la supervivencia de algún familiar), sobre todo si contemplan su retorno. Y estas dos dinámicas van a ampliar considerablemente la base de demandantes de servicios sociales por parte de los inmigrantes, también en sectores que, hasta la crisis, habían logrado acceder a posiciones sociales incluso de clase media.

El pronóstico del futuro crecimiento de las demandas no debe distorsionar la imagen real de los usuarios de los servicios sociales. Aunque existen estereotipos que presentan a los inmigrantes como usuarios crónicos, los estudios invalidan esa consideración (Moreno y Aierdi, 2008: 12 y s.). Los datos acreditan que la frecuencia con que usan los servicios sociales es inferior a su peso demográfico. No responden al tipo de usuario dependiente permanente de los servicios, que suele ser frecuente entre los usuarios autóctonos (Montagud y Torres, 2002: 22). Son usuarios satisfechos del trato que reciben, en pie de igualdad con los ciudadanos españoles (Calatrava y Marcu, 2006: 464).

Rodríguez Cabrero ha proporcionado importantes datos sobre la demanda de servicios por parte de los inmigrantes. Y la primera advertencia necesaria es que la estructura demográfica influye poderosamente; por ejemplo: la edad más joven de los inmigrantes genera menor demanda de servicios sanitarios, que se dispara a partir de los sesenta y cinco años. Y una segunda consideración se refiere al sector que debe servir de comparación: no es acertado comparar la demanda de los inmigrantes con la de la población total española; y si se compara con la población española pobre, los datos de Rodríguez Cabrero son contundentes: en torno al $49 \%$ de las familias españolas pobres o excluidas han recurrido alguna vez a los servicios sociales públicos o privados (Rodríguez Cabrero, 2003: 275; también Rodríguez Cabrero y Carrasco Carpio, 2006). Una cifra muy superior al acceso de los inmigrantes.

Los resultados de su estudio, realizado en base a una muestra exploratoria de 529 historias o ficheros de centros de servicios sociales, aportan información relevante. El estudio se llevó a cabo en Madrid, Barcelona, Málaga, Alicante y Pamplona. Una de las referencias importantes es el acceso a los servicios según la duración de su estancia en España. De todos ellos, el 40\% de los demandantes llevan residiendo aquí menos de un año; la cuarta parte reside desde hace más de dos años, y otra cuarta parte, entre uno y dos años. Las solicitudes 
más planteadas versan sobre la educación, debido a la elevada proporción de demandantes casados y en su mayoría con hijos. A continuación de las demandas educativas, hay otras cuatro con casi el mismo porcentaje, en torno al 16\%, que versan sobre vivienda, sanidad, empleo e información jurídica (Rodríguez Cabrero, 2003: 279-286).

Y no sólo los utilizan menos, sino que los utilizan de diferente manera que la población autóctona. Para los autóctonos, el sistema de servicios sociales «funciona a modo de última red de seguridad y de protección ante el riesgo de vulnerabilidad y de exclusión social», pero con los inmigrantes las cosas transcurren de otra manera. Para los inmigrantes, «los servicios sociales se convierten en la puerta de acceso para su proceso de inserción, más si cabe si tienen algún déficit con respecto al acceso al mercado laboral o la primera puerta de entrada en muchos casos para el resto de servicios públicos y de políticas sociales». Para los inmigrantes, por tanto, funcionan como primer trampolín de inserción, mientras que para el resto de la población es la última red de protección para evitar la exclusión (Moreno y Aierdi, 2008: 15-16).

La determinación de la evolución de la demanda de los inmigrantes a los servicios sociales se producirá como resultado de un saldo en el que intervienen dos elementos. Por una parte, en la práctica, los inmigrantes constituyen un sector social demandante, sobre todo en las primeras fases de su vida en España; la crisis económica y de empleo al reducir la llegada de inmigrantes hará reducir o detendrá el crecimiento de la demandas planteadas en los servicios sociales. Pero, por otra parte, el deterioro de la situación de empleo con sus secuelas salariales, de vivienda y de recursos para transferir a sus países de origen, hará aumentar las demandas. ¿¿Hacia donde se dirigirá el balance? La gravedad de la crisis y la presencia de un importante número de irregulares hace previsible el aumento de las demandas planteadas por los inmigrantes a los servicios sociales.

El crecimiento de la afluencia de inmigrantes a los servicios sociales va a generar la necesidad de reforzar o incrementar sus ofertas de traducción o intérpretes. Las necesidades serán más perentorias entre quienes lleven poco tiempo instalados en España y, por tanto, con menor aprendizaje del idioma. Tanto en la información personal como en la información escrita, será necesario proporcionar servicios con más frecuencia que hasta ahora en los numerosos idiomas de los inmigrantes. La necesidad de facilitar la comunicación requerirá el reforzamiento de la disponibilidad de expertos en diversos idiomas, accesibles con rapidez — en persona o por vía telefónica_- para que puedan trasladarse las peticiones y las demandas con la debida confidencialidad.

Otra dimensión que es necesario mencionar es que abordamos las demandas previsibles de inmigrantes, pero de quienes se encuentran en unas condiciones idóneas para realizar una actividad laboral plena. Esta delimitación excluye a aquellos que vienen a España en situaciones de discapacidad. Se trata de un grupo de población muy poco estudiado - y no solo en España- y cuyas necesidades y solicitudes son muy poco atendidas específicamente por las administraciones públicas. Un muy reciente informe sobre los inmigrantes 
con discapacidad, realizado por el CERMI para el Observatorio Permanente de la Inmigración, aporta las primeras perspectivas de un sector con graves necesidades.

Algo análogo ocurre con otro sector necesitado urgentemente de un tratamiento específico: los presos extranjeros. La Secretaria General de Instituciones Penitenciarias declaraba a la prensa, en junio de 2009, que el $35 \%$ de la población reclusa es de origen extranjero y una parte importante de él cumple condenas largas de prisión - de 9 años- por tráfico de drogas. La necesidad de establecer planes de acción específicos para esta población es urgente, sobre todo porque, además de su implicación en el tráfico de drogas, este colectivo puede acumular otras circunstancias que dificulten su adaptación a la salida de la cárcel. La falta de formación profesional, la larga estancia penitenciaria, el desarraigo en España, entre otras, dificultan su reinserción social posterior. Pero su reinserción viene condicionada por la experiencia carcelaria en la que el trabajo y el aprendizaje de una profesión desempeña un papel decisivo. La expectativa de una futura reinserción se fragua ante todo durante la etapa en la cárcel (Alós Moner et al., 2009).

El tratamiento de los internos en centros penitenciarios es uno de los que cuentan con mayores carencias, pese a la existencia de un servicio social penitenciario que interviene en la gestión de diferentes situaciones (libertad condicional, medidas de seguridad, trabajos en beneficio de la comunidad, etc.), pero, como los expertos destacan, su actividad no evita la intervención de la red pública de servicios sociales. Y aquí existe una de las mayores carencias. Como ha escrito Demetrio Casado (2008: 150):

El común de las comunidades autónomas, pese a su competencia exclusiva en servicios sociales, está siendo pasivo en la prevención de conductas delincuentes de quienes están en mayor riesgo de cometer delitos, como en las funciones de enlace facilitadoras de la reinserción pos-penitenciaria y, en su caso, para proveer recursos alternativos a la integración familiar.

La prostitución es una actividad con muy alta presencia de mujeres, y en menor medida de varones, inmigrantes, según todas las fuentes. La presencia de inmigrantes en la prostitución viene acompañada, la mayoría de las veces, de explotación por parte de mafias que, en muchas ocasiones, mantienen a estas personas en situaciones muy análogas a las de la esclavitud y con dependencia muy duradera para hacerles pagar los costes de su entrada, a menudo irregular, en España. Ningún país ha solucionado por completo esta explotación humana. Por eso mismo, se requiere reforzar las medidas de apoyo y las políticas de integración. La demanda de la prostitución puede reducirse por la crisis económica, con lo que estas políticas de integración pueden ser más oportunas, pero también más eficaces, en estos momentos. Aunque se trate de una explotación que requiere ser combatida en todo momento, el contexto de crisis puede convertir en muy conveniente un plan estratégico de integración social de estas personas. 
Pero la situación real es muy negativa. Los programas parecen orientados a situaciones a menudo coincidentes con la prostitución, como la toxicomanía, la violencia física contra la mujer o la persecución del proxeneta. Algunas entidades privadas vienen desempeñando un papel de asistencia y reinserción, con escasos apoyos económicos públicos: «Por lo demás, los servicios sociales públicos no cumplen con el papel que les corresponde en las carencias personales y la marginación social que padecen las personas en riesgo o situación de prostitución» (Casado, 2008: 151).

Las familias monoparentales han sido profusamente estudiadas en España, pero todavía no han merecido la misma atención las encabezadas por, sobre todo, mujeres inmigrantes. Al menos no disponemos todavía de estudios generales, como el abordado por Fernández Cordón y Tobío Soler (1999). Desde luego, no se conoce la magnitud real del fenómeno, que debe ser muy alto, superior sin duda al de los españoles. Y si debe ser superior en frecuencia, lo es también en gravedad y dificultades a que deben hacer frente. Los horarios laborales más dilatados de los inmigrantes, la duración de los desplazamientos, añadidos a las dificultades económicas y de inserción, convierten a las mujeres inmigrantes en un sector particularmente necesitado de estrategias específicas de inserción promovidas por los servicios sociales. La pérdida de empleo puede conducir a extensos sectores a situaciones de exclusión social, con el agravante de afectar a menores.

Precisamente otra circunstancia a considerar es la de los hijos. La inmigración — sea de varones, de mujeres o de parejas - presenta peculiaridades cuando involucra a sus hijos, en especial si son menores. Estos constituyen el sector estratégico para conocer el alcance de la integración real en cualquier sociedad, pero tampoco sus situaciones son homogéneas. Se distingue entre los hijos denominados de «segunda generación» — los hijos nacidos en el país de inmigración, en nuestro caso en España-y quienes llegaron con sus padres a edades tempranas y, por tanto, no han vivido en España toda su infancia ni su socialización primaria. Unas diferencias que, analizadas por Aparicio (2004), originan en nuestro país diferencias en las formas de inserción y en sus estrategias. La temprana integración escolar constituye desde luego un instrumento básico para la integración del menor (Foessa, 2008: 219). Pero los servicios sociales deben incrementar las medidas de apoyo, sobre todo a quienes no conocen el español, mediante las escuelas municipales de verano o refuerzos escolares a su aprendizaje.

En realidad, la referencia a los hijos implica una breve reflexión sobre la relación entre inmigración y familia. Se percibe, se analiza y se describe muy a menudo la inmigración como un fenómeno individual erróneamente. En la práctica, la inmigración es un hecho familiar o grupal, pero nunca estrictamente individual. Individual puede ser el desplazamiento material, pero su dinámica nunca lo es. Sus motivaciones son para el bienestar personal y familiar, los lugares de destino lo son por las conexiones personales, vecinales o familiares, que sirven como intermediarios de la operación. Es también el grupo quien primero acoge al recién llegado a su lugar de destino y se convierte en agente decisivo en su búsqueda de empleo. 
Todo este escenario se complica y se agrava cuando es el menor quien inmigra sin la presencia visible de su red familiar. La intervención de los servicios sociales deviene básica, no ya para su integración, sino para su mera supervivencia y alojamiento (López Azcona, 2008). La llegada de menores, además, está muy concentrada en determinadas provincias o comunidades autónomas, como en Canarias, a donde accede el mayor número de menores no acompañados. El número de menores implicados aumenta la gravedad objetiva del problema para los servicios sociales, muchas veces carentes de los recursos suficientes - humanos, económicos y de instalaciones- para hacer frente a un problema de esta magnitud y que requiere atención durante las veinticuatro horas del día. Parece obligado abordar un plan especial de atención e integración a los menores con el concurso de todas las administraciones, organizaciones no gubernamentales y el conjunto de la sociedad.

Pero, en último término, se requiere también programas y estrategias de acción en relación con la familia. Hay muchos indicios de que se necesita con urgencia respaldo de los servicios sociales y, en particular, de los especialistas en mediación.

El número de personas extranjeras involucradas en delitos de violencia de género es mayor de lo que le corresponde por su peso demográfico en España. La violencia de género afecta, además, a la posibilidad de adquirir el estatuto de refugiado (Arenas, 2008). Los datos acreditan que esta violencia es desproporcionadamente alta — si se compara con su peso demográfico en la población total- entre los extranjeros (no solo inmigrantes) en España. En el año 2008, el $37,1 \%$ de los agresores, y el $44,3 \%$ de las víctimas mortales por violencia de género eran extranjeras. Las medidas específicas de prevención son, desde luego, obligadas y urgentes.

Muchas de las condiciones de la emigración pueden deteriorar las relaciones de pareja: el choque cultural, la muy diferente posición social de la mujer en España en relación con la de sus países de origen, la convivencia frecuente de varias familias en una sola vivienda, las dificultades económicas y tantas otras pueden favorecer la ruptura de la pareja, cuando probablemente más importante es mantener la unidad económica para afrontar el proyecto migratorio. En cualquier caso, los datos disponibles requieren que se preste atención a la dimensión familiar de los inmigrantes como una estrategia específica de su integración. En este aspecto, como en el de las familias monoparentales, en el de la prostitución o en el de la violencia doméstica, se requieren asesoría jurídica y psicológica, casas de acogida, centros de día, pisos tutelados, residencias, albergues, comedores sociales, programas de formación profesional, acceso al empleo, etc.

Las dificultades se incrementan en el caso de hijos nacidos en países de habla no española y si llegan con déficits educativos graves en lectura o escritura. Estas circunstancias dificulta su inmediata adaptación e integración en España.

Las reflexiones anteriores están hechas desde el planteamiento actual de los servicios sociales, pero, considerado desde una dimensión dinámica, los 
servicios sociales incrementarán considerablemente sus actividades y sus destinatarios. Recientemente, Bernard Cazes (2009) planteaba explícitamente si no ha llegado la hora de ampliar sustantivamente las finalidades de las políticas sociales, y reclamaba, entre otras medidas, una reorientación para que incluya también a las políticas en favor de la "clase media olvidada", es decir, de varones y mujeres que ingresan salarios modestos.

Es preciso advertir que esta reorientación puede verse impulsada por vía de hecho. La situación de crisis económica mencionada comienza a generar dificultades de supervivencia en amplios sectores, desconocidos hasta ahora para los servicios sociales. Sectores de la baja clase media pueden convertirse en sus usuarios y demandantes de apoyo en situación de crisis económica. Por consiguiente, la reorientación de las políticas sociales puede producirse, no como resultado de una opción ideológica o política, sino como consecuencia de la agudización de la crisis y la afluencia de nuevos sectores sociales. Y la situación ofrece panoramas muy graves ya en junio de 2009. En esa fecha, el ministro de Trabajo informó que el $25 \%$ de las familias con todos sus integrantes en paro no cobran ningún tipo de prestación social. Cuando se deja de cobrar la prestación contributiva o asistencial por desempleo, entra en este caso en juego el ingreso mínimo de solidaridad, también conocido como «salario social». En cualquier caso, ya existe un sector importante de nuestra sociedad con necesidades de supervivencia y convertido en potencial demandante objetivo de los servicios sociales.

Esta reorientación puede producirse como consecuencia de las necesidades del conjunto de la población, pero de forma más frecuente va a suceder entre la población inmigrante. Sectores que han logrado posiciones sociales intermedias o se han abierto camino con empresas o negocios de diversos tipos, pueden encontrarse con graves dificultades a corto plazo. Por consiguiente, inmigrantes de clase media pueden ser los nuevos demandantes de los servicios sociales, al resultar víctimas de crisis.

\section{Recursos sociales para el bienestar social de los inmigrantes}

La creciente llegada y diversidad de la población inmigrante ha generado retos para nuestro sistema de bienestar, de los que deriva el planteamiento de, al menos, tres objetivos:

1. Garantizar el acceso y la integración en las diferentes áreas de prestaciones y servicios.

2. Adaptar el sistema y sus profesionales a la pluralidad de culturas y nuevas necesidades.

3. Innovar, tanto en la intervención social, como en la creación de centros y recursos especializados, entre ellos, los de acogida y convivencia intercultural.

Los servicios sociales son puerta de entrada, al tiempo que apoyo transversal, para el resto de las áreas de bienestar, desde el especial peso de los 
servicios sociales primarios en la acogida, hasta la colaboración con el sistema educativo, sanitario y la mediación para la participación en los mercados de trabajo y vivienda. Un enfoque que se traslada desde la asistencia social hacia la integración y la interculturalidad.

\subsection{Servicios y recursos de acogida}

El proceso de acogida abarca todas aquellas medidas de apoyo destinadas a dotar a las personas inmigrantes de los conocimientos y las habilidades necesarias para desenvolverse autónomamente en la sociedad y tener acceso, en igualdad con los ciudadanos autóctonos, a los recursos y a los servicios sociales de carácter general (PECIDEF, 2007: 199). Se trata de un concepto integral en el que, al conocimiento del idioma, de los valores y de las normas de la sociedad que recibe, se suma la información sobre los derechos y los deberes de los que hacen del nuestro, su nuevo país.

No existe un itinerario normalizado del proceso de acogida. Sus características, dispositivos y recursos implicados variarán, entre otros, según los siguientes criterios:

1. Según el perfil y la situación de la persona inmigrante. Será necesario tener en cuenta si se trata de una migración temporal o estable, la situación administrativa en que se encuentra — dado que la falta de documentación puede convertirse en un obstáculo en el acceso a los recursos públicos- y la propia proximidad cultural y de idioma que facilita el acercamiento a los recursos del sistema.

2. La implicación de los actores públicos y privados y la configuración propia generada por un sistema que pivota principalmente en la colaboración entre comunidades autónomas y corporaciones locales. Además, la necesidad de dar una respuesta rápida, flexible e innovadora a la llegada de la inmigración ha implicado de forma determinante a la iniciativa social, tanto a través de organizaciones tradicionalmente asistenciales, como de nuevas organizaciones especializadas. La red Acoge o SOS Racismo fueron referencias pioneras en el sector.

3. La historia del fenómeno migratorio en el área receptora. Los primeros recursos de que se disponen son los relacionados con el alojamiento temporal y la cobertura de las necesidades más inmediatas para ir ampliando progresivamente el itinerario a medida que la migración adquiere un carácter más estable.

Dentro de la intervención en acogida, existen situaciones de especial vulnerabilidad que requieren de una atención específica. Es el caso de las personas migrantes que llegan de forma irregular a través de las rutas del Estrecho y de África a nuestras costas. La primera respuesta es la del rescate y la asistencia humanitaria, en que, de forma directa, colaboran las organizaciones no gubernamentales, entre ellas, Cruz Roja. Una vez atendidas las primeras necesidades, 
las personas que llegan a través de estas vías irregulares pasan a ser alojadas en centros de estancia o acogida temporal, en los que participan en diversos programas de atención mientras que se dirime su situación de permanencia en nuestro país. En el caso de los menores no acompañados, la trayectoria de intervención los dirige hacia centros y recursos especializados vinculados al sistema de protección de menores.

La intervención de asilo y refugio o de migración programada requiere de una atención de acogida diferencial. En el caso de los trabajadores y las trabajadoras temporeros en campañas agrícolas o en obra o servicio determinado, la temporalidad de su estancia condiciona la respuesta de acogida. Pueden darse al menos dos tipos de situaciones: la de trabajo temporal con retorno al país de origen o bien la inclusión en un ciclo migratorio interno que depende de la estacionalidad de los trabajos agrícolas. La campaña de recogida de la aceituna en el interior andaluz, de la fresa en Huelva o de los cultivos hortofrutícolas en Murcia o Lérida forman parte de este ciclo. Desde la perspectiva de los servicios sociales, deberá tenerse en cuenta que la temporalidad puede limitar a unos pocos meses la intervención en un mismo lugar.

Para atender a estas situaciones distintas, las administraciones locales, con el apoyo de la Administración general y autonómica, han venido vertebrando diversos recursos de acogida, entre ellos, oficinas de información, albergues temporales y la intervención a través de la extensión y consolidación del perfil de mediador cultural que desempeña tareas, tanto en la relación del inmigrante con el sistema de bienestar, como en la mediación en el mercado laboral y el acceso a la vivienda.

En materia de acogida, la colaboración entre la Administración General del Estado y las comunidades autónomas se articula a través del Fondo de Apoyo a la Acogida y la Integración de Inmigrantes, así como del refuerzo educativo de los mismos. Creado en 2005, apoya como líneas prioritarias:

a) El desarrollo de la red de acogida integral, que incluye programas de orientación social y laboral y plazas de alojamiento y manutención para inmigrantes en situación de vulnerabilidad.

b) El apoyo a servicios de información, orientación y acompañamiento, clases de idiomas, asesoría jurídica, traducción e interpretación, derivación a servicios de carácter general, formación ocupacional e inserción social y laboral. El Fondo prevé una mayor asignación económica para aquellas comunidades que presentan una mayor presión demográfica, caso de Andalucía, Cataluña, Comunidad Valenciana, Madrid, Murcia, Ceuta, Melilla y Canarias. En el caso de esta última comunidad, la llegada de inmigración procedente de África occidental a través de «cayucos» ha hecho necesario un especial refuerzo de los dispositivos de acogida, tanto estables como de emergencia, así como de la atención y escolarización de menores no acompañados. El Ministerio de Trabajo e Inmigración apoya el desarrollo del portal web integralocal.es en el que se encuentran disponibles para consulta información y experiencias de acogida desarrollados en el ámbito local. 
Junto a la intervención pública, la participación de la iniciativa social —y, dentro de ella, las propias asociaciones de inmigrantes-y de los agentes sociales (empresarios y sindicatos) desempeña un importante papel. Conviene recordar que, en el caso de los sindicatos, realizan actuaciones de preacogida a través de la información y la formación sobre derechos y deberes de los trabajadores en el marco de la organización del contingente. Por su parte, el empresariado debe asegurar como mínimo el alojamiento a los trabajadores contratados.

El Plan Estratégico de Ciudadanía e Integración establece como objetivos la aplicación de programas introductorios a la sociedad de acogida a través de actuaciones homologadas y la articulación de una red estatal de dispositivos de acogida integral en todo el territorio nacional.

\subsection{Empleo y formación}

La participación de los inmigrantes en el mercado de trabajo aporta dinamismo a nuestra economía, al tiempo que estabilidad al sistema de seguridad social. Si el trabajo es clave para el desarrollo de la persona, en el caso de los inmigrantes no comunitarios, esta afirmación cobra, si cabe aún, mayor trascendencia, especialmente en el primer momento de estancia en nuestro país, en el que el empleo justifica la renovación de la residencia y se convierte en garantía de recursos mínimos en un sistema de protección social que, al carecer de cotizaciones, limita el acceso a las prestaciones del sistema contributivo.

En nuestro país, la potenciación de la contratación en origen constituye el principal objetivo de la coordinación entre mercado de trabajo e inmigración. El contingente y la puesta en marcha del Catálogo de Ocupaciones de Difícil Cobertura hacen operativa esta línea. El Gobierno aprueba, con carácter anual, por acuerdo del Consejo de Ministros, un contingente de trabajadores extranjeros que permite la contratación programada de trabajadores que no se hallan ni residen en España, llamados a desempeñar empleos con vocación de estabilidad y que son seleccionados en sus países de origen a partir de las ofertas presentadas por los empresarios. El contingente comprende:

- Una cifra provisional, así como las características de las ofertas de empleo de carácter estable para un año natural, que puedan ser cubiertas a través de este procedimiento.

- Un grupo de visados para búsqueda de empleo dirigidos a hijos o nietos de español de origen.

- Un número de visados limitados a determinados sectores de actividad u ocupaciones en un ámbito territorial concreto.

Los visados permiten la estancia para búsqueda de trabajo en nuestro país durante tres meses. Igualmente, regula particularidades en el procedimiento de contratación de trabajadores de temporada, así como la gestión de ofertas para personas concretas que, con carácter estable, quieran establecer una empresa. 
Los empresarios presentan las solicitudes, personalmente o a través de su representante legal (que, para este caso, podrán ser las organizaciones empresariales), y pueden también participar, directa o indirectamente, en los procesos de selección en origen de los trabajadores. Pueden desarrollarse cursos de formación, en España o en los países de origen, dirigidos a los trabajadores seleccionados o preseleccionados.

El visado concedido por la autoridad consular incorporará la autorización inicial de residencia y trabajo por cuenta ajena de un año (con carácter general) de duración, contado desde la fecha en que se efectúe la entrada en España. Dicha autorización está limitada a un ámbito territorial y a un sector de actividad determinados, y permite la incorporación inmediata de los trabajadores a la empresa, así como su afiliación y/o su alta en la seguridad social. En el plazo de un mes desde su entrada en España, los trabajadores deben solicitar personalmente la tarjeta de identidad de extranjero. Junto a este procedimiento, el Ministerio de Trabajo e Inmigración cuenta con la Unidad de Grandes Empresas, dirigida a los empresarios que quieran incorporar trabajadores extranjeros de alta cualificación.

La distribución de los trabajadores inmigrantes por sectores laborales es distinta a la del resto de trabajadores. El sector que mayor número de extranjeros ocupa en España es la construcción, le sigue la hostelería, el empleo doméstico, el comercio y la agricultura. A finales de 2007, los extranjeros suponían el $19,7 \%$ del total de los trabajadores de la construcción, el 24,4\% del total de los trabajadores de la hostelería y el 11,6\% de los empleados y las empleadas del hogar (Pajares, 2007). Este último sector, mayoritariamente ocupado por mujeres, va más allá del cuidado del hogar para ir al cuidado de las personas. En este sentido, se establece una vinculación directa con las medidas de profesionalización de los sistemas de atención a la dependencia.

No obstante, y a pesar de esta participación de los trabajadores inmigrantes, aún existen desigualdades en el acceso y en la permanencia en el mercado laboral que justifican diversas acciones y programas de intervención, entre ellos:

- Programas que tengan por objeto desarrollar acciones tendentes a la integración en el mercado de trabajo de los inmigrantes a través del desarrollo de itinerarios integrados de inserción laboral individualizados.

- Fomento entre la población inmigrante de la iniciativa empresarial y el trabajo por cuenta propia o trabajo asociado (autoempleo, microcréditos y economía social).

- Refuerzo de la presencia de inmigrantes en programas desarrollados en el marco de las políticas activas de empleo y de la colaboración con entidades de intermediación laboral.

- Información y formación sobre prevención de riesgos laborales y promoción de la seguridad y la salud en el trabajo.

- Programas que tengan por objeto la erradicación de la trata de personas con fines de explotación laboral o sexual y que promuevan su inserción en el mercado de trabajo. 
- Programas que posibiliten la inserción social y laboral de familias inmigrantes en zonas rurales con baja densidad de población, a través de procesos personalizados de selección, formación y acompañamiento.

- Programas de creación de servicios que ofrezcan información, asesoramiento, acompañamiento, traducción de documentos y otros trámites necesarios para la homologación de títulos y la convalidación de estudios realizados en los países de origen, así como otras acciones de apoyo para la diversificación profesional.

\subsection{Asistencia sanitaria}

La salud constituye un derecho básico y universal para todos los ciudadanos. No obstante, esta afirmación tiene sus particularidades. La normativa reconoce el derecho a la asistencia sanitaria de los extranjeros en las mismas condiciones que los españoles, en caso de estar empadronados, de ser menores de edad o en caso de embarazo (durante el embarazo, el parto y el posparto), además del derecho a la asistencia sanitaria pública de urgencias al contraer enfermedades graves o accidentes, cualquiera sea su causa, para todos, sin limitación alguna, y a la continuidad de dicha atención hasta la situación de alta médica.

El sistema de salud debe garantizar el acceso de los inmigrantes y la adecuación mutua entre servicios y nuevas necesidades apostando por la integración, pero teniendo también en cuenta las particularidades que presenta la diversidad cultural. Persisten distintos tipos de obstáculos en el acceso (Ortega Córdoba e Izquierdo Díaz, 2007):

a) Las barreras normativas y administrativas, entendidas como las especiales dificultades que puede atravesar esta población para acogerse oficialmente a los derechos fundamentales de los que goza la población autóctona.

b) Las barreras lingüísticas y de comunicación, especialmente evidentes en las personas extranjeras procedentes de países de habla diferente al español.

c) Las barreras culturales y religiosas, especialmente relevantes entre los extranjeros procedentes de países islámicos y entre los procedentes de países con un sistema de protección social y sanitario muy diferente al nuestro.

d) El rechazo pasivo del sistema o de los profesionales sanitarios. La atención sanitaria a esta población, como ya se ha dicho, tiene una especial complejidad. A esto se le une la falta de formación de los profesionales en la atención a la diversidad cultural y en la atención de enfermedades no comunes en nuestro medio. Todos son factores que agravan el riesgo de exclusión de los extranjeros (selección adversa) de cualquier sistema sanitario público.

El sistema sanitario ha previsto una tarjeta sanitaria individual para los extranjeros residentes que les abre el acceso a las prestaciones sanitarias del Sistema Nacional de Salud. El problema persiste en aquellos no empadronados y/o indocumentados. Con todo, varias comunidades autónomas, como Andalucía, Valencia, Navarra y Madrid, han articulado medidas que permiten 
acceder a la sanidad pública a las personas en situación de exclusión social que no tienen acceso a la tarjeta sanitaria y que padecen problemas de salud. En el caso andaluz, al desarrollo de la tarjeta se une la carpeta que se entrega al inmigrante, sea regular o irregular, que contiene información sobre los servicios sanitarios públicos de Andalucía en varios idiomas, así como una historia clínica portátil, útil para que los profesionales que le atienden puedan anotar las actuaciones sobre la salud individual del inmigrante, lo cual permite su continuidad allí donde se encuentre.

El enfoque del sistema pasa por el paso de la asistencia a la atención, incorporando los programas de prevención y promoción de la salud y la capacitación a los profesionales de la salud para una mejor atención al emigrado y a sus familias. En el caso de las mujeres, habría que añadir la vertiente de la salud reproductiva y sexual, así como la especial vigilancia de las situaciones de riesgo de violencia de género y prácticas culturales que atentan contra su integridad, caso de la ablación genital.

\subsection{Centros y recursos dirigidos a la población inmigrante}

La situación administrativa de la persona inmigrante, el momento en que se encuentra en el nuevo itinerario en nuestro país y la situación de vulnerabilidad especial han dado lugar a la creación de centros especializados. A continuación, tratamos de ofrecer una tipología de estos centros y recursos.

a) Centros de internamiento. Los centros de internamiento, dependientes del Ministerio del Interior, están previstos para las situaciones en que las personas inmigrantes se encuentran a la espera de retorno, devolución o procedimiento preferente de expulsión en los casos establecidos por la ley. La situación de ingreso por retorno se puede producir bien en frontera, bien por encontrarse en situación irregular en nuestro país, y siempre que la resolución de retorno no pueda ejecutarse en setenta y dos horas. El ingreso por causa de expulsión será dictado por el juez como consecuencia de la implicación en causa judicial por infracciones graves a la seguridad del Estado, el orden público o tráfico e inmigración clandestina. En cualquier caso, la duración máxima del internamiento no podrá exceder los cuarenta días.

La ley establece que los lugares de internamiento para extranjeros no tendrán carácter penitenciario, y estarán dotados de servicios sociales, jurídicos, culturales y sanitarios. De igual forma, la ley también reconoce los derechos y los deberes de las personas internadas. Entre ellos, se encuentran los siguientes: recibir asistencia médica y sanitaria adecuada, ser asistidos por los servicios de asistencia social del centro y contar con intérprete y abogado de oficio. Los menores extranjeros no acompañados no podrán ser ingresados en estos centros.

Conviene señalar que la orden de devolución puede quedar en suspenso cuando se trate de mujeres embarazadas o cuando se formalice una solicitud de asilo hasta que se haya decidido sobre su admisión o inadmisión a trámite. Para estas situaciones específicas, se cuenta con recursos distintos. 
b) Las oficinas de extranjeros tratan de informar y de normalizar la situación administrativa de la persona inmigrante en los aspectos de residencia y trabajo. La puesta en marcha de estas oficinas ha permitido aunar en un solo centro administrativo los procedimientos que antes eran iniciados en distintas sedes (policía, administración de trabajo, etc.). Las oficinas están ubicadas en la capital de provincia y dependerán orgánicamente de la Delegación o Subdelegación del Gobierno.

c) Centros de migraciones. La red de Centros de Migraciones del Ministerio de Trabajo e Inmigración, recogida en el reglamento, está integrada por los Centros de Acogida a Refugiados (CEAR), los Centros de Estancia Temporal de Inmigrantes (CETI) de Ceuta y Melilla, y, en su caso, centros de nueva creación. Los centros desarrollan tareas de información, atención, acogida, intervención social, formación o, si procede, derivación hacia otros recursos especializados. Los Centros de Estancia Temporal de Inmigrantes (CETI) de Ceuta y Melilla ofrecen servicios y prestaciones sociales básicas al colectivo de inmigrantes y solicitantes de asilo que llegan a alguna de las ciudades autónomas. Más abajo haremos referencia especial a los CEAR.

d) Centros de acogida temporal. Puestos en marcha principalmente por las comunidades autónomas y las administraciones locales, pretenden cubrir las necesidades primarias (alojamiento, manutención, información y orientación) de los inmigrantes en situación de emergencia o vulnerabilidad social.

La Administración local tiene muchas competencias y responsabilidades en materia de acogida que pueden favorecer la integración de los inmigrantes: empadronamiento, servicios de información, acceso a servicios sociales primarios, reagrupación familiar, arraigo social, etc.

Una de las primeras respuestas que se ofrecen desde el ámbito local es la de la información y la acogida temporal, bien por parte de la Administración local o en colaboración con la iniciativa social. Se trata de servicios coyunturales, con un tope máximo de días o meses de atención, para evitar la cronificación de los usuarios y que ofrecen información, asesoramiento y derivación. En el caso de inmigrantes temporeros, algunas administraciones locales han institucionalizado redes de recursos de acogida. Sirva como ejemplo ilustrativo el caso de la provincia de Jaén, con cerca de una veintena de albergues en red y un programa de mediación intercultural asociado que se intensifica como dispositivo durante la campaña de recogida de aceituna.

e) Centros de apoyo a la integración y participación de los inmigrantes

Los centros de apoyo a la integración son dispositivos de ayuda de segundo nivel, que complementan la atención social básica de servicios sociales de atención primaria. Tienen como objetivos: apoyar la intervención técnica de los equipos de servicios sociales generales, prestar atención social de emergencia y para inmigrantes en riesgo de exclusión y, con cada vez mayor frecuencia, ofrecer servicios de mediación intercultural y sociolaboral. 
Los centros de participación e integración de inmigrantes son recursos públicos de acceso directo que representan a diferentes nacionalidades y que tienen como finalidad la promoción del bienestar de la población inmigrante y autóctona, a través de actuaciones que favorezcan procesos de convivencia intercultural que contribuyan al conocimiento mutuo, el respeto y la tolerancia.

Por último, incluimos una breve referencia al caso de los menores no acompañados. Una vez determinada la edad en colaboración con los servicios sanitarios, el menor o la menor será atendido por los servicios de protección de menores. El principio que prima es el de máximo interés y reagrupación familiar del menor, de forma que, una vez oído al menor y a los servicios de protección, se resuelve sobre su repatriación al lugar de origen, aquel en que se encuentren sus familiares, o sobre su permanencia en España. En el caso de menores tutelados por entidades de protección de menores, y una vez alcanzada la mayoría de edad y hayan participado adecuadamente en las acciones formativas y actividades programadas para su integración, se podrá recomendar autorización temporal de residencia. Progresivamente, se van extendiendo programas y centros de apoyo para el tránsito hacia una vida fuera del centro que les permita continuar sus planes educativos, así como su proceso de integración e inserción en nuestro país.

\section{Conclusiones}

La presencia de un 10\% de la población inmigrante en España obliga a mejorar los servicios y las prestaciones destinados a afrontar sus necesidades, que, en muchos casos, son específicas respecto al conjunto de la población. Ello requiere incrementar los recursos destinados a favorecer su integración real, imprescindible para mantener los niveles de cohesión social que exige nuestra constitución. Pero no basta con incrementar los recursos económicos y materiales, se necesita igualmente potenciar otras actuaciones de gestión y organización. Algunas es preciso mencionarlas:

1. Ensanchar los objetivos $-\mathrm{y}$, por consiguiente, las dotaciones humanas e instrumentales - de los servicios sociales. Desde las primeras leyes de servicios sociales, ha cambiado profundamente la sociedad española y sus necesidades. En general, sus objetivos se encuentran en exceso circunscritos a las diversas manifestaciones de la pobreza y la exclusión. Desgraciadamente, estas realidades subsisten, pero los servicios sociales deben atender también a nuevas demandas surgidas después de unas décadas de desarrollo económico. Las que plantean los inmigrantes deberán ser un sector prioritario, porque muchas veces acumulan más necesidades que la población española.

Ese ensanchamiento de los objetivos tiene que materializarse con el reforzamiento de la cooperación entre las distintas administraciones y con las organizaciones no gubernamentales, pero que atienden a necesidades 
sociales. La intensificación de las relaciones con otras dependencias administrativas y servicios, como los de empleo, redundará en beneficio de los administrados.

2. Activar el enfoque proactivo. Los servicios sociales están diseñados normativamente para atender a aquellos que acuden a demandar información y ayuda. Esa orientación es superada en la práctica por el esfuerzo de los profesionales, que realizan su actividad de forma más activa en la mayoría de los casos. Se trata de insertar el enfoque proactivo en la base misma de la existencia y organización de los servicios. Demetrio Casado ha destacado con acierto esta nueva orientación que requiere actividades de carácter preventivo y la gestión con sentido anticipatorio de las prestaciones. Para él, cuando los servicios sociales o, en general, las políticas sociales no actúan anticipada o tempranamente, son los más necesitados, los menos informados o los menos capaces quienes se llevan la peor parte:

Si la intervención y las políticas sociales no actúan pro-activamente, su oferta llegará menos de lo deseable a personas afectadas [...]. Para cumplir su misión, al igual que otras ramas de recursos pro bienestar, los servicios sociales están llamados a gestionarse con anticipación o prontitud, saliendo al encuentro de las demandas latentes, sin esperar necesariamente a que las mismas lleguen a manifestarse plenamente. (Casado: 284-285)

Esa "pro-actividad» no puede quedar restringida únicamente a las funciones específicas de los servicios sociales. Se necesita proyectarla, igualmente, a otros servicios públicos de los cuales los inmigrantes están muy necesitados. La educación, la vivienda, el empleo, la sanidad, los procesos administrativos de residencia, etc. tienen que ser incorporados habitualmente a los procedimientos de actuación de los servicios sociales.

3. Mejorar la coordinación de la red de servicios y equipamientos con recursos disponibles para atender a la población inmigrante. La coordinación resulta imprescindible entre las diferentes ramas de la Administración: central, autonómica y local. Se necesita una mayor coordinación de sus servicios, como de sus proyectos e innovaciones para la buena utilización de los recursos escasos. Pero todavía es más urgente la coordinación con las múltiples acciones de la iniciativa privada y dirigidas a ayudar a los inmigrantes. La multitud de normas e iniciativas que actúan promoviendo la integración de los inmigrantes hace imprescindible organizar la cooperación y la coordinación de acciones.

Es un hecho incuestionable que la iniciativa privada cuenta con un papel creciente en los servicios sociales. De hecho, las primeras leyes autonómicas contemplaban de manera muy limitada la presencia de la iniciativa privada (Alemán Bracho y García Serrano, 1997), y con cierta desconfianza. Pero desde entonces, con o sin ánimo de lucro, la presencia de la iniciativa privada se ha incrementado considerablemente. Por eso, se ha propuesto que: 
[...] sería conveniente caminar hacia la consideración de las entidades privadas como plenamente colaboradoras, en un sentido similar, por poner un ejemplo, al que existe en la Seguridad Social, donde compañías de seguros puramente privadas fueron catalogadas desde el comienzo como colaboradoras en la gestión del sistema; eso sí, con todo el control y exigencias que el sector público precisa. Otro modelo en el que poder inspirarse es el sistema educativo, en el que los centros concertados forman parte integrante y colaboradora, de aquél. (Alonso Seco, 2007: 396)

4. La aportación humana es esencial para el adecuado funcionamiento de los servicios sociales en su atención a los inmigrantes, pero es necesario mejorar los instrumentos informativos. De manera concreta, se necesita incrementar sustancialmente el uso de las nuevas tecnologías. Múltiples informaciones, comunicaciones y apoyos disponibles pueden y deben ser accesibles en la red. Cada vez más, Internet es un instrumento conocido y empleado por los inmigrantes, y no hay razón para excluir esta materia. Pero no se trata de multiplicar hasta el infinito los centros que proporcionan esa información. Lo deseable es organizar una web que aglutine, integre y sintetice las informaciones adecuadas de manera clara para personas que no conocen la estructura institucional y administrativa de España. Y todo ello resolviendo la gran diversidad de lenguas de los demandantes de información.

5. La necesidad de aumentar la información estadistica disponible sobre la inmigración y los servicios sociales. Ningún plan ni ninguna estrategia puede elaborarse certeramente sin el conocimiento preciso de la realidad a la que se dirige. Y si es cierto que se han mejorado las informaciones disponibles sobre la inmigración, muy poco se conoce sobre las demandas realmente planteadas por los inmigrantes en las diferentes comunidades autónomas. Y sin saber lo que está pasando, sin duda será difícil planear respuestas acertadas a las nuevas demandas sociales.

En todo caso, es indudable que los servicios sociales afrontan un desafío importante con la atención a los inmigrantes, y no lo hacen en las mejores condiciones. Como han escrito Guillén et al. (2001: 219), expertos conocedores de la situación:

[...] los Servicios Sociales reaccionan a posteriori, pero, sobre todo, mediante respuestas improvisadas y urgentes, ni siquiera cotejadas con otras experiencias muy positivas como las elaboradas en otros países europeos. El resultado es que, una vez paridos los planes estratégicos, lo hacen sobre un entramado socio-asistencial disperso en iniciativas y no ideado para ello. Y lo que es más, sobre una base de recursos y medios muy vulnerables.

Si a ello se une la situación de crisis económica, la conclusión evidente es el serio desafío que habrán de afrontar los servicios sociales en nuestro futuro inmediato. 


\section{Referencias bibliográficas}

Alemán Bracho, C. (1995). «La política de asistencia social desde la transición democrática». Revista de Estudios Políticos, 87.

Alemán Bracho, C. et al. (2009). Las políticas de integración social de los inmigrantes en las CCAA españolas. Madrid: Fundación BBVA.

Alemán Bracho, C. y García Serrano, M. (1997). "Política social e iniciativa social». En: Alemán Bracho, C. y Garcés Ferrer, J. (ed.). Política Social. Madrid. McGrawHill.

- (2008). Los servicios sociales especializados en España. Madrid: R. Areces.

Alonso Seco, J.M. (2007). «Mejoras jurídico-institucionales en los Servicios Sociales». En: Casado, D. y Fantova, F. (ed.). Perfeccionamiento de los servicios sociales en España. Madrid: Fundación Foessa, 373-413.

Alós Moner, R.; Martín Artiles, A.; Miguélez, F. y Gibert, F. (2009). «¿Sirve el trabajo penitenciario para la reinserción?: Un estudio a partir de las opiniones de los presos de las cárceles de Cataluña». Revista Española de Investigaciones Sociológicas, $127,1133$.

Andrés Juste, P. (2004). «Inmigración y derechos sociales: Una introducción al marco legal de los servicios sociales para los inmigrantes». Revista de Derecho Migratorio y Extranjería, noviembre, 267-295.

Aparicio, R. (2004). «El proceso de integración de los hijos de los inmigrantes». En: $2^{\circ}$ Congreso La Familia en la Sociedad del Siglo XXI. Madrid. FAD, 84-99.

Arenas Hidalgo, N. (2008). «La violencia doméstica y la posibilidad de adquirir el estatuto de refugiado: Derecho comunitario y práctica española». Derecho Migratorio y Extranjería, 17, 59-103.

Calatrava, A. y Marcu, S. (2006). «El acceso de los inmigrantes a los servicios sociales en la Comunidad de Madrid, como factor de integración». Estudios Geográficos, 261, 441-470.

Casado, D. (2007). "Objetivos permanentes y enfoques de los Servicios Sociales». En: Casado, D. y Fantova, F. (ed.). Perfeccionamiento de los Servicios Sociales en España. Madrid: Fundación Foessa, 255-289.

- (2008). «Los Servicios Sociales: carencias relativas a las situaciones de pobreza, marginación social, exclusión y desigualdades». En: Fundación Foessa. VI Informe sobre Exclusión y Desarrollo Social en España 2008. Conclusiones, 145-156.

Cazes, B. (2009). «Preface». En: Damon, J. Questions Sociales: analyses anglo saxonnes. Socialement incorrect? París: PUF.

Fernández Cordón, J.A. y Tobío Soler, C. (1999). Las familias monoparentales en España. Madrid: Ministerio de Trabajo y Asuntos Sociales.

Fundación Foessa (2008). VI Informe sobre Exclusión y Desarrollo Social en España 2008. Conclusiones. Madrid.

Guillén, E.; Lucas, F. de; Pérez, D. y Arias, A. (2001). «Servicios sociales e inmigración: límites y retos para una nueva política social». Alternativas: Cuaderno de Trabajo Social, 9, 211-239.

López Azcona, A. (2008). «El tratamiento de los menores no acompañados en el Derecho Español». Derecho Migratorio y Extranjería, 18, 103-137.

Montagud, X. y Torres, F. (2002). «Inmigración y Servicios Sociales. Entre el estereotipo y la realidad». Cuadernos Electrónicos de Filosofía del Derecho, 6, 35 p., www.uv.es/CEFD.

Moreno Márquez, G. y Aierdi Urraza, X. (2008). "Inmigración y Servicios Sociales: ¿última red o primer trampolín?». Zerbitzuan, 44, diciembre, 719. 
PECIDEF (2007). Plan Estratégico Ciudadanía e Integración. Madrid: Ministerio de Asuntos Sociales.

Rodríguez Cabrero, G. (2003). «Protección Social de los Inmigrantes Extranjeros». En: Izquierdo, A. (ed.). Inmigración: Mercado de Trabajo y Protección Social en España. Madrid. Consejo Económico y Social de España, 249-301.

Rodríguez Cabrero, G. y Carrasco Carpio, C. (2006). «El acceso de los extranjeros a los servicios públicos y las prestaciones sociales». En: Aja, E. y Arango, J. (eds.). Veinte años de inmigración en España: Perspectivas jurídica y sociológica. Barcelona: Fundación CIDOB.

Soriano, R. (2004). «Administración pública e inmigración en España». En: TriniDAD, A. (ed.). Administración pública y Estado de bienestar. Madrid. Thompson Cívitas, 337-359. 